\title{
Dynamic Modeling and Analysis of Buck Converter based Solar PV Charge Controller for Improved MPPT Performance
}

\author{
Venkatramanan D, Student Member, IEEE \\ Department of Electrical Engineering \\ Indian Institute of Science (IISc) \\ Bangalore, India \\ email: venkat86ram@gmail.com
}

\begin{abstract}
Maximum power point tracking (MPPT) control is a key functionality in solar photo-voltaic (PV) based power generation systems that enhances the efficacy of energy extraction. A pulse-width modulated (PWM) power converter connected to the $\mathrm{PV}$ is designed to perform this essential function. A variety of MPPT control schemes are available in literature, many of which are voltage based techniques wherein the PV bus voltage is controlled in closed loop to the required level that achieves MPP tracking. However, a suitable plant model of the PV fed converter system that facilitates the design of such a PV voltage control loop for MPPT purpose, is not fully covered in literature. In this paper, a small-signal model is developed for a buck converter based charger system fed by a PV source, which is a nonlinear active source. Based on this model, the relevant transferfunctions are analytically derived. The control to converter input voltage transfer-function $\widetilde{v}_{i n} / \widetilde{d}$ thus obtained, is useful in the systematic design of voltage controller bandwidth, that facilitates the selection of perturbation period of a typical perturb \& observe $(\mathbf{P} \& \mathbf{O})$ MPPT controller. Such an approach for control design is verified in this work on a PV system with energy storage, which is typically used in dual-mode or standalone PV applications. The P\&O MPPT control is validated on a buck PV charge controller system employing a $36 \mathrm{~V}$ battery bank. Experiments conducted on a $1 \mathrm{~kW}$ hardware prototype verify the accuracy of the proposed analytical transfer-functions and the performance of the PV-charger system.
\end{abstract}

Keywords-Solar PV, battery charging, MPPT, buck converter modeling, frequency response analysis, control transfer-function.

\section{INTRODUCTION}

Solar Photo-voltaic (PV) power generation systems have garnered significant attention recently spurred by the growing need to meet energy requirements with clean-energy resources [1]-[3]. A pulse-width modulated (PWM) power converter is invariably required to interface PV with the grid in case of a grid-tied system, or with the battery bank in case of a dual-mode or standalone PV charger system [4]-[6]. Maximum power point tracking (MPPT) control is an essential feature incorporated in these systems that enhances the efficacy of energy extraction. A variety of MPPT control schemes are available in literature and in many of these techniques, PV bus voltage is chosen as the target variable that is controlled in closed loop to the required level to ensure MPP tracking [7][9].

While a wide body of work exists on MPPT algorithms along with their comparative study and implementation chal-

This work was supported by CPRI, Ministry of Power, Government of India, under the project Power conversion, control, and protection technologies for microgrid.

978-1-5386-9316-2/18/\$31.00 (c) 2018 IEEE

\author{
Vinod John, Senior Member, IEEE \\ Department of Electrical Engineering \\ Indian Institute of Science (IISc) \\ Bangalore, India \\ email: vjohn@iisc.ac.in
}

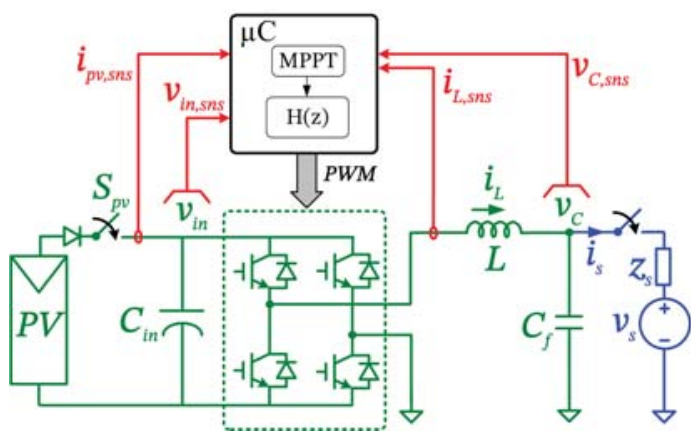

Fig. 1: Power circuit schematic of buck converter based solar PV power generation system.

lenges [8], [10], literature is rather limited on plant modeling of the PV system that captures the dynamics of dc bus voltage directly fed by PV as a function of the converter duty-ratio, $\widetilde{v}_{i n} / \tilde{d}$, which is essential for PWM converter control that performs the MPPT function. Often steady-state relationships for converter control are used assuming MPPT speed is much slower than converter bandwidth. In other words, it is typically assumed that the converter dynamics are much faster than the MPPT control loop bandwidth. [11] suggests a model for SEPIC converter based PV system that incorporates peakcurrent mode control, while [7] proposes a second-order plant for a boost-converter based PV system. In [12], a buck-boost converter model is employed for PV panel characterization. A buck voltage source converter is a widely prevalent topology in single-stage grid-tied PV inverters and dual-mode PV systems with battery storage [9]. It is essential to model the control to PV voltage transfer-function to obtain the best performance from the buck PV system functioning in closed-loop control.

In this paper, a small-signal model for a buck converter based PV-charger system is developed based on simplified linear models of solar PV source and battery, using which relevant transfer-functions are obtained. In particular, the control to PV input voltage transfer-function $\widetilde{v}_{i n} / \widetilde{d}$ serves as the plant for closed-loop voltage control of the converter in a typical voltage-based perturb \& observe ( $\mathrm{P} \& \mathrm{O})$ MPPT scheme [8]. The settling time of the voltage-loop, that is governed by the voltage controller bandwidth, determines the minimum timelatency required between consecutive perturbations in the $\mathrm{P} \& \mathrm{O}$ algorithm for accurate tracking performance. Selection of the perturbation period below the minimum latency value causes erroneous power measurement since the system transients are not settled, and this leads to degraded MPPT performance. Thus, the transfer-functions analysis carried out here facilitate a systematic design of nested MPPT controller functioning 


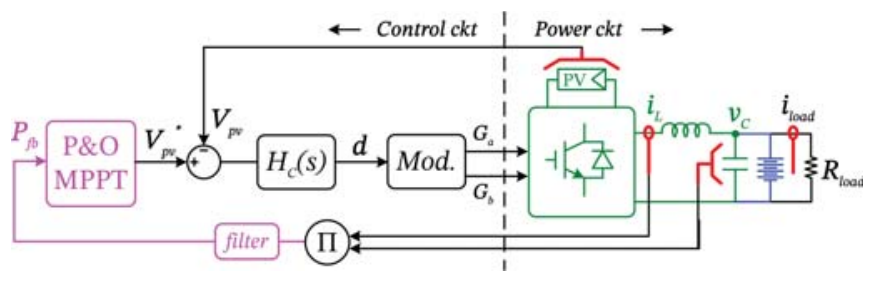

Fig. 2: Circuit schematic of a single-loop voltage based $\mathrm{P} \& \mathrm{O}$ MPPT control scheme for a buck solar PV charge controller.

TABLE I: Buck PV charge-controller ratings and parameters

\begin{tabular}{|c|c|}
\hline Item & Value \\
\hline Converter rating & $1 \mathrm{kVA}$ \\
\hline PV rating & $900 \mathrm{~W}$ \\
\hline Nom. PV voltage $v_{i n}$ & $120 \mathrm{~V}$ \\
\hline Battery rating $v_{B}, C_{A H}$ & $36 \mathrm{~V}, 60 \mathrm{Ah}$ \\
\hline DC capacitor $C_{i n}, r_{e s r}$ & $1230 \mu F, 80 \mathrm{~m} \Omega$ \\
\hline Switching frequency $f_{s w}$ & $20 \mathrm{kHz}$ \\
\hline Filter inductor $L, r_{L}$ & $0.8 \mathrm{mH}, 20 \mathrm{~m} \Omega$ \\
\hline Filter capacitor $C_{f}$ & $60 \mu F$ \\
\hline
\end{tabular}

in closed-loop. The suggested procedure for transfer-function analysis is generic and can be applied for PV systems with other converter types as well. A test system based on a frequency response analyzer is suggested for active converter transfer-function measurements during operation. Such a setup is suitable for impedance characterization of PV strings as well. A P\&O MPPT algorithm based on [7], [13], that uses PV voltage as the control variable is designed for the PV system to validate the proposed transfer-function analysis.

Experimental results are furnished for a $1 \mathrm{~kW}$ buck PV charge controller prototype that charges a $36 \mathrm{~V}$ battery bank. The power circuit schematic of the PV system considered is shown in Fig. 1. The analytically obtained and the measured transfer-functions show a close match in the desired frequency range. These experimental results verify the accuracy of the analytical transfer-functions expressions and the performance of the PV-charger system.

\section{SYSTEM DESCRIPTION}

The power circuit of the PV system considered in this work comprises of a $\mathrm{H}$-bridge buck converter interfacing PV with a power source $v_{s}$ as shown in Fig. 1, the ratings of which are listed in Table. I. The parameter $z_{s}$ represents the source impedance of $v_{s}$. The dc-bus is fed directly by the PV source through a series blocking diode. The converter is interfaced through an LC-filter to the source $v_{s}$, which is a $36 \mathrm{~V}$ Leadacid battery bank in this case. It is pointed out that the second power source $v_{s}$ can be a battery, or utility grid as in a typical grid-tied PV inverter system. In this paper, a buck converter based PV-charger for battery banks is considered for study. The control circuit schematic of the single-loop voltage based $\mathrm{P} \& \mathrm{O}$ MPPT algorithm employed in this work is shown in Fig. 2.

\section{SMALL-SIGNAL MODEL}

In this section, a small-signal dynamic model of the buck PV-charger system is developed based on simplified linear models of solar PV source and battery. The frequency range of interest for control is $1 / 10^{t h}$ of the switching frequency $f_{s w}$, which corresponds to $2 \mathrm{kHz}$ in this case based on the system parameters in Table. I. The simplified models considered in this work are such that they capture the dominant characteristics of the actual system accurately in this frequency range and hence are adequate for analysis and controller design.

1) PV source model: Typically for PV array modeling, system analysis and MPPT design, static single-diode circuit model such as that indicated in Fig. 3(a) is considered [14][16]. However, based on the insight gathered from the physical structure of the PV panel, it can noted that the PV also inherently carries dynamic storage elements that are not captured in the static model. These are in the form of - (a) diffusion capacitance $C_{d}$ due to the forward-biased semiconductor junction of the PV cells, (b) transition capacitance $C_{t}$ due the reverse-biased junction of the bypass diode connected across the PV panel, and (c) equivalent series inductance $L_{s}$ caused by the meander-type metalized wire tabbing interconnects joining various cells in series. These parasitics elements need to be considered in the source model for comprehensive system analysis and switching converter controller design. The linear dynamic model of the PV source suggested in this work, that includes the effects of dynamic elements and is suitable for small-signal analysis with switching converters, is shown in Fig. 3(b). Here, $r_{d}$ represents the total dynamic resistance of the forward-biased PV diode at a given operating point, and $R_{s}$ represents the resistance of the metallic interconnects.

Based on the small-signal bode-plot measurements conducted on the PV panel HSTBF24300P, that is employed in this work which is rated for $300 \mathrm{~W}, 36 \mathrm{~V}$ at MPP [17], it is observed that the effect of poles and zeros caused by the parasitic storage elements come into effect only beyond the frequency range of interest of $2 \mathrm{kHz}$. Thus, the smallsignal Thevenin's model of the PV source considered for system analysis and converter control, is that of a resistance $R_{p v}=R_{p}+R_{s}$, where $R_{p}=\left(r_{d} \| R_{s h}\right)$ as indicated in Fig. 3(c). In this work, three panels are connected in series yielding a power of $900 \mathrm{~W}$ at MPP as indicated in Table. I. The value of $V_{p v}$ equals the open-circuit PV voltage of $120 \mathrm{~V}$, and $R_{p v}$ is measured to be equal to $2.2 \Omega$.

2) Battery model: The frequency response measurements conducted on the $36 \mathrm{~V}$ battery bank using Solartron CellTest impedance analyzer, at two levels of input excitation, indicate that only the real part of the battery impedance is dominant, as shown in Fig.4. Hence, the battery bank is modeled as a voltage source $V_{B}$ with a series resistance $r_{B}=30 \mathrm{~m} \Omega$.

3) Cable model: The $200 \mathrm{~m}$ interconnecting cable from rooftop PV to the power converter is modeled as a resistance and denoted as $r_{c a b}=0.6 \Omega$. The twisted-pair power cable inductance is found to be negligibly small.

\section{A. Transfer-function Analysis}

The validity of these simplifying assumptions is ascertained through a comparative study of transfer-functions obtained from analysis and experimentation. For the buck converter system indicated in Fig. 1, the proposed DC and small-signal AC equivalent circuit models based on the canonical model development discussed in [18], are shown in Fig. 5(a) and (b) 


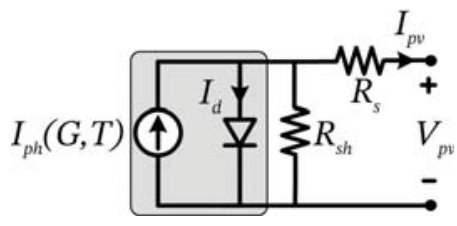

(a)

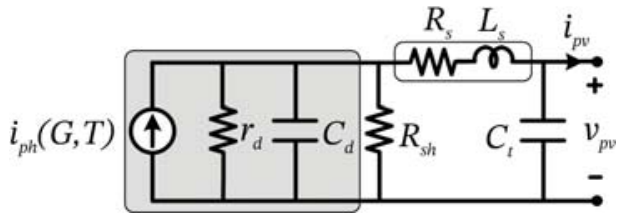

(b)

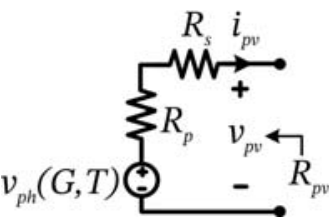

(c)

Fig. 3: Solar PV source equivalent circuit models showing (a) conventionally used static model, (b) linear dynamic model for small-signal analysis with switching converters, (c) simplified linear model.
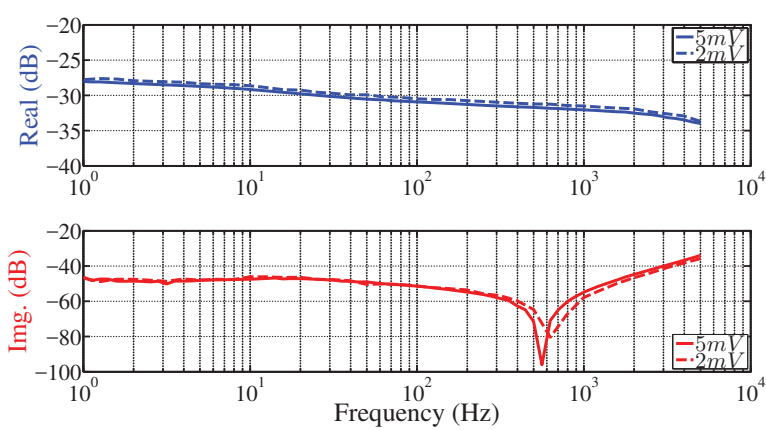

Fig. 4: Measured battery impedance characteristics using Solartron impedance analyzer.

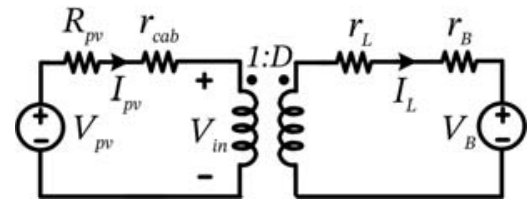

(a)

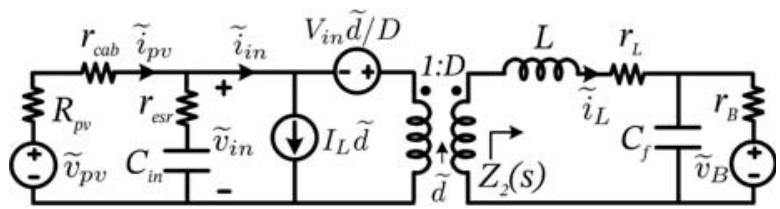

(b)

Fig. 5: Proposed dynamic model of PV based buck-converter system showing (a) DC model, and (b) small-signal AC model.

respectively. From the DC model, the steady-state operating points of circuit quantities for a quiescent duty-ratio $D$ are given by,

$$
\begin{gathered}
R_{1}=R_{p v}+r_{c a b} \\
R_{2}=r_{L}+r_{B} \\
I_{p v}=D I_{L} \\
\Rightarrow I_{L}=\frac{D V_{p v}-V_{B}}{D^{2} R_{1}+R_{2}} \\
V_{i n}=\left(V_{p v}-I_{p v} R_{1}\right)
\end{gathered}
$$

From the small-signal AC model in the frequency range of interest, it follows that,

$$
\begin{gathered}
Z_{2}(s)=s L+r_{L}+\frac{r_{B}}{1+s C_{f} r_{B}} \approx s L+r_{L}+r_{B} \\
\Rightarrow Z_{2}(s)=s L+R_{2}
\end{gathered}
$$

From the values of $r_{B}$ and $C_{f}$ indicated in Table. I, the pole introduced by their combination in output load impedance $Z_{2}(s)$ in (6) is around $90 \mathrm{kHz}$, which is much higher than the switching frequency $f_{s w}$. Hence, its effect is ignored for design calculations. Also, for the calculation of converter control transfer-functions of the PV-charger system, the disturbances in PV voltage $\widetilde{v}_{p v}$ and battery voltage $\widetilde{v}_{B}$ are considered to be zero. Hence, $\widetilde{v}_{p v}=0$ and $\widetilde{v}_{B}=0$.

For a given disturbance input $\tilde{d}$ in the converter duty-ratio, the resulting disturbance in inductor-current is given by,

$$
\begin{aligned}
\widetilde{i}_{L} & =\left(\frac{V_{i n}-I_{p v} R_{1}}{D^{2} R_{1}+Z_{2}}\right) \widetilde{d} \\
\Rightarrow \frac{\widetilde{i}_{L}}{\widetilde{d}} & =\left(\frac{V_{i n}-I_{p v} R_{1}}{D^{2} R_{1}+R_{2}+s L}\right)
\end{aligned}
$$

The resulting disturbance in input current owing to $\widetilde{d}$ and $\widetilde{i}_{L}$ can be computed as,

$$
\begin{gathered}
\widetilde{i}_{i n}=D \widetilde{i}_{L}+I_{L} \widetilde{d} \\
\frac{\widetilde{i}_{i n}}{\widetilde{d}}=D\left(\frac{\widetilde{i}_{L}}{\widetilde{d}}\right)+I_{L}
\end{gathered}
$$

Using (4) and (8) in (10), we get

$$
\begin{gathered}
\Rightarrow \frac{\widetilde{i}_{i n}}{\widetilde{d}}=D\left(\frac{V_{i n}-I_{p v} R_{1}}{D^{2} R_{1}+R_{2}+s L}\right)+\frac{D V_{p v}-V_{B}}{D^{2} R_{1}+R_{2}} \\
\Rightarrow \frac{\widetilde{i}_{i n}}{\widetilde{d}}=\frac{D V_{i n}-D I_{p v} R_{1}+\left(D V_{p v}-V_{B}\right)\left[1+s L /\left(D^{2} R_{1}+R_{2}\right)\right]}{\left(D^{2} R_{1}+R_{2}\right)\left[1+s L /\left(D^{2} R_{1}+R_{2}\right)\right]} \\
\Rightarrow \frac{\widetilde{i}_{i n}}{\widetilde{d}}=\frac{2 D V_{i n}-V_{B}+s L\left(D V_{p v}-V_{B}\right) /\left(D^{2} R_{1}+R_{2}\right)}{\left(D^{2} R_{1}+R_{2}\right)\left[1+s L /\left(D^{2} R_{1}+R_{2}\right)\right]}
\end{gathered}
$$

From the input current disturbance $\widetilde{i}_{i n}$, the resultant input dcbus voltage disturbance $\widetilde{v}_{i n}$ can be computed as,

$$
\begin{array}{r}
\frac{\widetilde{v}_{i n}}{\widetilde{d}}=-\left[R_{1} \|\left(r_{e s r}+\frac{1}{s C_{i n}}\right)\right]\left(\frac{\widetilde{i}_{i n}}{\widetilde{d}}\right) \\
\Rightarrow \frac{\widetilde{v}_{i n}}{\widetilde{d}}=-R_{1}\left[\frac{1+r_{e s r} C_{i n} s}{1+\left(R_{1}+r_{e s r}\right) C_{i n} s}\right]\left(\frac{\widetilde{i}_{i n}}{\widetilde{d}}\right) \\
\text { and, } \quad \frac{\widetilde{i}_{p v}}{\widetilde{d}}=\left[\frac{1+r_{e s r} C_{i n} s}{1+\left(R_{1}+r_{e s r}\right) C_{i n} s}\right]\left(\frac{\widetilde{i}_{i n}}{\widetilde{d}}\right)
\end{array}
$$




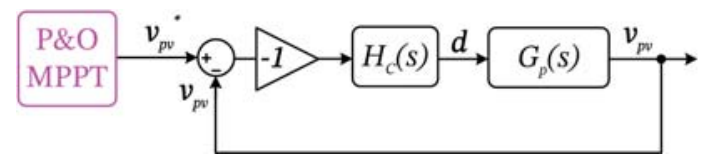

Fig. 6: Small-signal model of single-loop voltage based P\&O MPPT control architecture.

Equation (8) that relates the inductor current $\widetilde{i}_{L}$ with dutyratio is further simplified and re-written in canonical form as,

$$
\frac{\widetilde{i}_{L}}{\widetilde{d}}=k_{d c, i}\left[\frac{1}{1+s / \omega_{p 1}}\right]
$$

Similarly, (14) that relates input voltage $\widetilde{v}_{\text {in }}$ dynamics with duty-ratio is further simplified and re-written in canonical form as,

$$
\frac{\widetilde{v}_{i n}}{\widetilde{d}}=k_{d c, v}\left[\frac{\left(1+s / \omega_{z 1}\right)\left(1+s / \omega_{z 2}\right)}{\left(1+s / \omega_{p 1}\right)\left(1+s / \omega_{p 2}\right)}\right]
$$

where,

$$
\begin{gathered}
k_{d c, i}=\frac{V_{p v}-2 I_{p v} R_{1}}{D^{2} R_{1}+R_{2}} \\
k_{d c, v}=-R_{1}\left(\frac{2 D V_{i n}-V_{B}}{D^{2} R_{1}+R_{2}}\right) \\
\omega_{p 1}=\frac{D^{2} R_{1}+R_{2}}{L} \\
\omega_{p 2}=\frac{1}{R_{1} C_{i n}} \\
\omega_{z 1}=\omega_{p 1}\left(\frac{2 D V_{i n}-V_{B}}{D V_{p v}-V_{B}}\right) \\
\omega_{z 2}=\frac{1}{r_{e s r} C_{i n}}
\end{gathered}
$$

\section{B. Discussion}

The dc gain of the inductor current transfer-function $\widetilde{i}_{L} / \tilde{d}$ in (16) is governed only by the equivalent series resistances in the buck-charger circuit through an inverse relationship. And since these resistances are typically quite small in value, the resultant dc gain in (16) is large. Thus, the case of a battery charger is distinct from a traditional voltage controlled buckconverter, where the dc gain is governed by the load resistance that is typically much larger than the series resistances in a well designed system.

The input bus voltage transfer function $\widetilde{v}_{i n} / \widetilde{d}$ in (17) carries a negative dc gain as indicated in (19), along with two distinct poles. One is caused by the pole of the output filter inductor $L$, and the other due to the corner frequency of the input filter capacitor $C_{i n}$. This suggests that in a PV based buck-charger, the converter input voltage dynamics are not governed purely by the input filter, but are coupled to the output side as well.

\section{Controller Design}

The evaluation of plant gains, time-constants and settling times in terms of system parameters and quiescent operating point are carried out using (16) and (17), which thus facilitate the design of voltage loop for the MPPT controller. Fig. 2 shows the overall control architecture of a P\&O MPPT scheme based on single-loop voltage control. The MPPT block dictates the set-point of input dc-bus voltage, and the voltage controller $H_{c}(s)$, which is chosen to be a PI-controller, tracks the dc reference by suitably adjusting the control duty-ratio $d$. The corresponding small-signal model of the control loop is shown in Fig. 6.

$$
\begin{gathered}
G_{p}=\frac{\widetilde{v}_{i n}}{\widetilde{d}} \\
H_{c}=k_{p}\left(\frac{1+s / \omega_{c}}{s / \omega_{c}}\right) \\
G_{c}=\frac{T}{1+T}=\frac{-G_{p} H_{c}}{1-G_{p} H_{c}}
\end{gathered}
$$

Since the plant $G_{p}$ carries a negative dc gain, an inverting unity gain block needs to be present in the forward path to offset this effect and ensure stable closed-loop converter operation. This is seen in Fig. 6 and (26).

\section{Transfer-Function Measurement}

\section{A. Setup Description}

The setup for converter transfer-function measurements consists of the buck-converter, as described in Section I, and a N4L PSM3750 frequency response analyzer (FRA). The two are interfaced through a TMS28377S DSP based digital controller as shown in Fig. 7, that provides the PWM pulses for the power converter control.

\section{B. Test Procedure}

Transfer-function measurements are done when the buckconverter is operating in open loop at a quiescent point governed by the constant duty $D$ provided by the controller, which is set to an appropriate value that sets the other quiescent variables $I_{L}, I_{p v}$ and $V_{p v}$. The FRA instrument provides a frequency-sweep sinusoidal output signal $v_{o}$, which is sampled by the ADC of the DSP and used as the duty-ratio disturbance input $\widetilde{d}$ as illustrated in Fig. 7. This is added to the $D$ inside the controller that generates the total duty command $d_{t o t}$. The FRA performs transfer-function measurement by sweeping $v_{o}$ across the chosen frequency range and evaluating the ratio of its Channel-B input $C h_{B}$ to Channel-A input $C h_{A}$.

$$
\begin{gathered}
d_{\text {tot }}=D+\widetilde{d} \\
G_{\text {meas }}(s)=\frac{C h_{B}(s)}{C h_{A}(s)}
\end{gathered}
$$

The input $C h_{A}$ is set permanently to $v_{o}$ which corresponds to the duty-ratio disturbance $\widetilde{d}$, while $C h_{B}$ is selected among $\widetilde{i}_{L}$, $\widetilde{i}_{p v}$ and $\widetilde{v}_{i n}$ which are fed to the FRA through appropriate sensors. The FRA thus measures the required transfer-functions indicated in (16) and (17). The duty $\widetilde{d}$ is swept from $1 \mathrm{~Hz}$ to $5 k H z$, and its peak amplitude is set to be $|\widetilde{d}|=0.0015$. This disturbance magnitude is adequately small such that the linear approximations of the buck-converter system are valid, and the measured disturbance variables are sinusoidal in nature although the PV input is a non-linear source. 


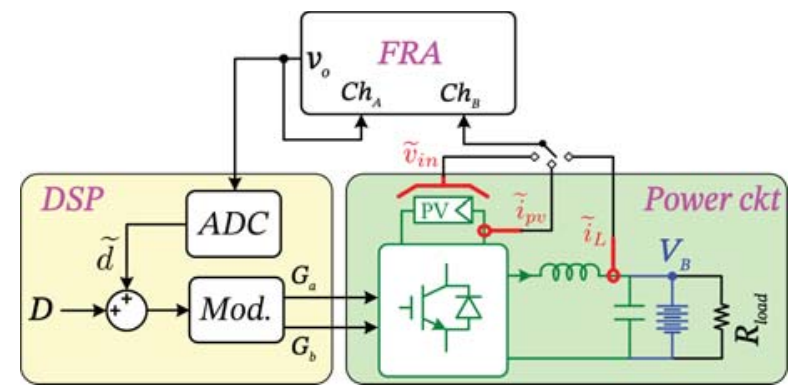

Fig. 7: Converter transfer-function measurement scheme using N4L frequency response analyzer and DSP.

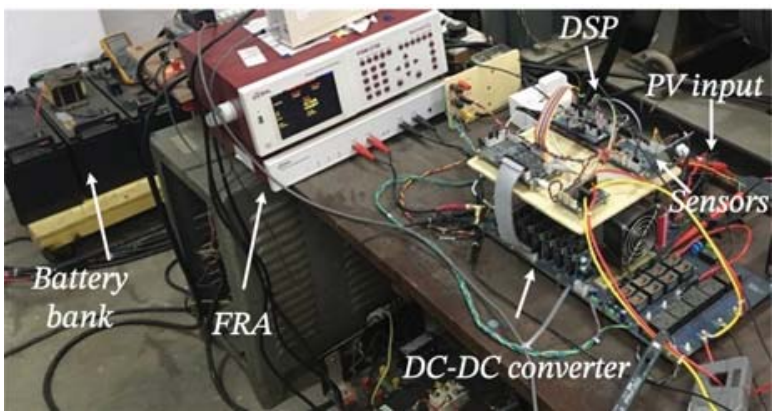

Fig. 8: Experimental setup showing network analyzer, buck PV charger controller system and battery bank.

\section{EXPERIMENTAL VALIDATION AND DISCUSSION}

Fig. 8 shows the hardware setup consisting of the power converter, battery bank, DSP and the FRA instrument. Fig. 9 shows the measured disturbances $\widetilde{v}_{i n}$ and $\widetilde{i}_{p v}$ for a step-change in amplitude of the $10 \mathrm{~Hz}$ sinusoidal duty-ratio disturbance command $\widetilde{d}$ from DSP. In this test, the amplitude $|\widetilde{d}|$ is reduced from 0.005 to 0.0025 . It can be seen that when $|\widetilde{d}|$ $=0.005$, the response in $\widetilde{v}_{i n}$ and $\widetilde{i}_{p v}$ are non-sinusoidal in nature, indicating that the applied disturbance input magnitude is not adequately small for linear approximations to be valid in the system. However, with reduced amplitude of $|\widetilde{d}|=$ 0.0025 , the responses in $\widetilde{v}_{i n}$ and $\widetilde{i}_{p v}$ are more sinusoidal. Hence, for the FRA characterization, an amplitude of $|\widetilde{d}|=$ 0.0015 is chosen as indicated in Section V-B, that ensures

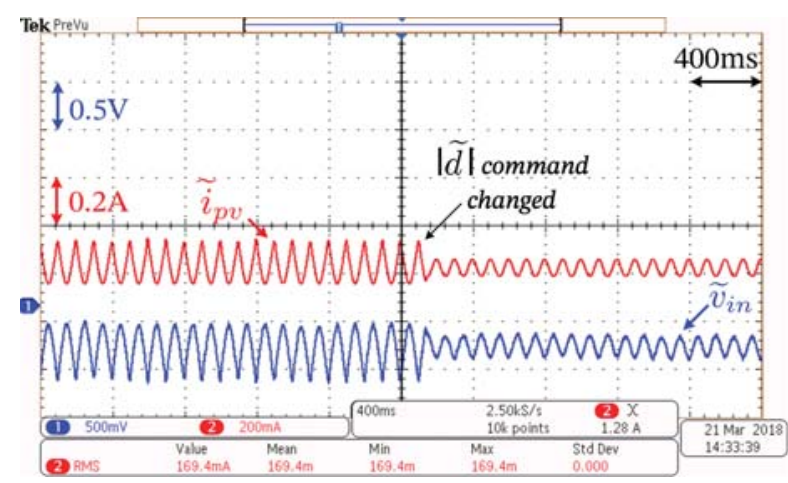

Fig. 9: Illustration of non-linear nature of PV fed buckconverter showing measured disturbances in $\widetilde{i}_{p v}$ and $\widetilde{v}_{\text {in }}$ for different magnitudes of injected duty-ratio disturbance $\widetilde{d}$.
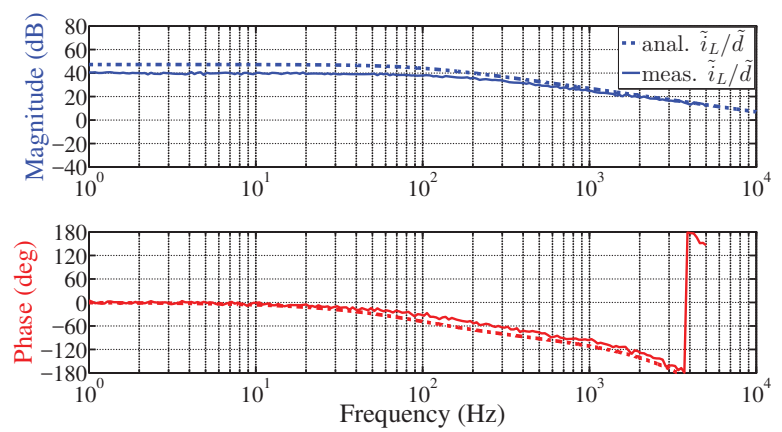

(a)
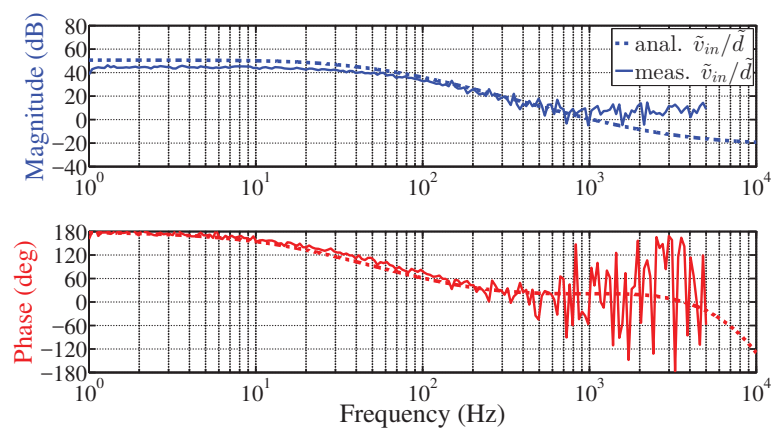

(b)

Fig. 10: Comparison of analytical and experimentally measured converter frequency response characteristics showing (a) inductor current transfer-function $\widetilde{i}_{L} / \widetilde{d}$, (b) PV voltage transfer-function $\widetilde{v}_{i n} / \widetilde{d}$.

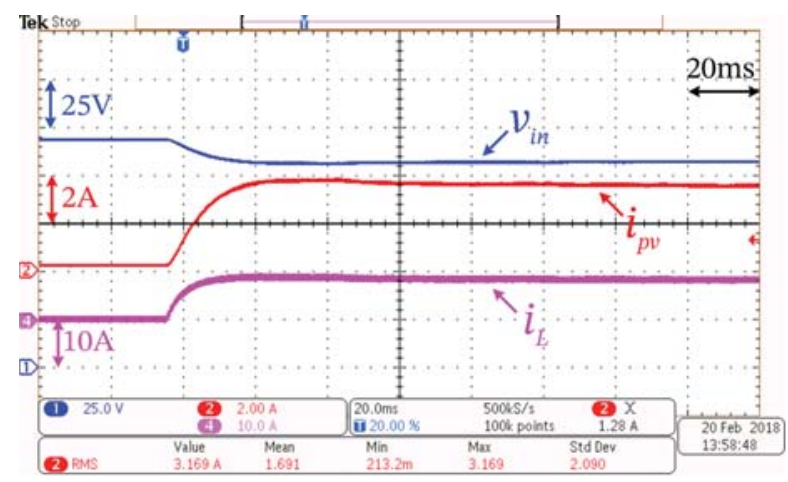

Fig. 11: Responses of $i_{L}, v_{i n}$ and $i_{p v}$ for a step-command change in $D$ from 0.305 to 0.345 .

that linear approximations in the converter are valid during measurements. The parameter variation in the buck-converter system due to temperature change has been neglected in the analysis.

Fig. 10 compares the analytically obtained transferfunctions in (16) and (17) with that of the measured frequency response characteristics when the converter is in open-loop operation for $D=0.305$, and $I_{L}=0.5 \mathrm{~A}$. It can be seen that there is a close match between the two results. The $\widetilde{i}_{L} / \widetilde{d}$ transfer-function in Fig. 10(a) is seen to have a first-order pole at around $100 \mathrm{~Hz}$, with the phase crossing over $180^{\circ}$ at around $4 \mathrm{kHz}$. This is due to the loop-delay function $e^{-1.5 s T_{d}}$ that has been included while plotting the bode-plot of (16). The transfer-function $\widetilde{v}_{i n} / \widetilde{d}$ in Fig. 10(b) is observed to be an 


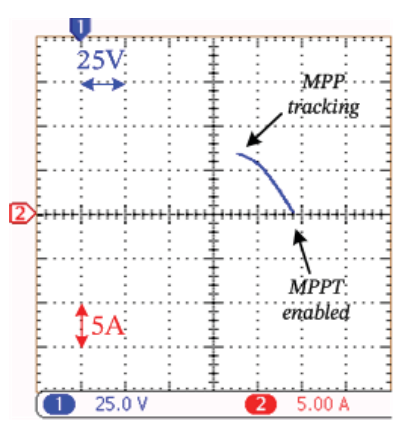

(a)

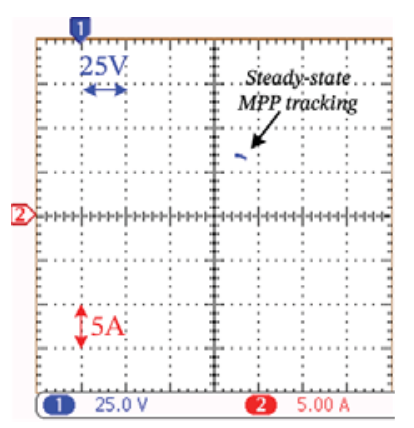

(b)
Fig. 12: Experimental converter performance for P\&O MPPT control in $I-V$ characteristics of the PV string showing (a) startup characteristics during MPPT enable, and (b) steadystate tracking characteristics.

over-damped second-order system with two distinct poles at around $30 \mathrm{~Hz}$ and $100 \mathrm{~Hz}$. The effect of zeros, which are distinct second-order in nature, come into effect only after $1 \mathrm{kHz}$. The deviations observed in measurements in Fig. 10 above $1 \mathrm{kHz}$ are due to the extremely low levels of signals beyond the measuring range of the FRA instrument.

Fig. 11 shows the responses of $i_{L}, v_{i n}$ and $i_{p v}$ for a stepchange is quiescent duty-command $D$ from 0.305 to 0.345 , when the converter is operating in open-loop. It can be noticed that $i_{L}$ exhibits a first-order step response that is faster than the over-damped second-order responses manifested by $v_{i n}$ and $i_{p v}$. The settling times of the system variables are in consonance with that predicted by the expressions in (16)-(17) as well as the measured transfer-functions in Fig. 10. This verifies the accuracy of the proposed small-signal model of the system.

Using (16)-(17), a PI-controller for closed-loop control of $\mathrm{PV}$ bus voltage is designed using which a voltage-based $\mathrm{P} \& \mathrm{O}$ MPPT scheme is implemented. The settling times of the system response correspond to the expected closed-loop system bandwidth, and is used to specify the minimum possible update rate to be employed for the $\mathrm{P} \& \mathrm{O}$ algorithm. The corresponding startup and stead-state performance of the MPPT control are indicated in Fig. 12(a) and (b).

\section{CONCLUSION}

In this paper, a small-signal linear model for a buckconverter based PV power generation system is proposed based on simplified linear models of battery and PV source. The PV bus voltage transfer-function $\widetilde{v}_{i n} / \widetilde{d}$ thus derived is useful in the systematic design of a voltage-controller, that determines the settling time of the system for a given perturbation in voltage command. This value is critical in selecting the perturbation period of a voltage based P\&O MPPT control scheme. A test system using a frequency response analyzer is suggested that facilitates the measurement of the relevant converter transferfunctions during operation. The P\&O MPPT scheme designed based on this analysis is implemented on a $1 \mathrm{~kW}$ buck PV charge controller prototype for validation. Experimental results and transfer-function measurements performed on the converter verify the accuracy of the proposed model and the performance of the overall system during open loop and closed-loop MPPT operation. Such an analytical model is useful in systematic design of MPPT control loops in singlestage PV systems with battery storage.

\section{REFERENCES}

[1] S. S. Nag, S. Mandal, and S. Mishra, "Solar pv based dc power supply for rural homes with analog, multiplier-less mppt controller," in Proc. 43rd Annu. Conf. IEEE Indus. Electron. Soc., Oct 2017, pp. 2689-2694.

[2] J. Amanor-Boadu, E. Sanchez-Sinencio, and M. W. Asmah, "A universal fast battery charging and management solution for stand-alone solar photovoltaic home systems in sub-saharan africa," in Proc. IEEE Power Energy Soc. PowerAfrica Conf., June 2017, pp. 174-179.

[3] A. Poullikkas, "A comparative assessment of net metering and feed in tariff schemes for residential PV systems," Sustainable Energy Technol. Assessments, vol. 3, pp. 1-8, Sept 2013.

[4] D. Venkatramanan and V. John, "Integrated higher-order pulse-width modulation filter-transformer structure for single-phase static compensator," IET Power Electron., vol. 6, no. 1, pp. 67-77, Jan 2013.

[5] S. Dutta, D. Debnath, and K. Chatterjee, "A grid-connected single-phase transformerless inverter controlling two solar pv arrays operating under different atmospheric conditions," IEEE Trans. Ind. Electron., vol. 65, no. 1, pp. 374-385, Jan 2018.

[6] A. Hintz, U. R. Prasanna, and K. Rajashekara, "Comparative study of the three-phase grid-connected inverter sharing unbalanced three-phase and/or single-phase systems," IEEE Trans. Ind. Appl., vol. 52, no. 6, pp. 5156-5164, Nov 2016.

[7] N. Femia, G. Petrone, G. Spagnuolo, and M. Vitelli, "Optimization of perturb and observe maximum power point tracking method," IEEE Trans. Power Electron., vol. 20, no. 4, pp. 963-973, July 2005.

[8] _- Power electronics and control techniques for maximum energy harvesting in photovoltaic systems. CRC press, 2017.

[9] R. Teodorescu, M. Liserre, P. Rodriguez, and F. Blaabjerg, Grid Converters for Photovoltaic and Wind Power Systems. John Wiley \& Sons, 2011, vol. 29.

[10] M. A. G. de Brito, L. Galotto, L. P. Sampaio, G. d. A. e Melo, and C. A. Canesin, "Evaluation of the main mpt techniques for photovoltaic applications," IEEE Trans. Indus. Electron., vol. 60, no. 3, pp. 11561167, March 2013.

[11] S. J. Chiang, H. J. Shieh, and M. C. Chen, "Modeling and control of pv charger system with sepic converter," IEEE Trans. Ind. Electron., vol. 56, no. 11, pp. 4344-4353, Nov 2009.

[12] P. Bharadwaj and V. John, "High performance buck-boost converter based pv characterisation set-up," in Proc. IEEE Energy Convers. Congr. Expo. (ECCE), Oct 2017, pp. 4425-4432.

[13] T. Esram and P. L. Chapman, "Comparison of photovoltaic array maximum power point tracking techniques," IEEE Transactions on Energy Conversion, vol. 22, no. 2, pp. 439-449, June 2007.

[14] B. Pallavi and V. John, "Linearised model for pv panel power output variation with changes in ambient conditions," Sädhanā, vol. 42 , no. 12 , pp. 2183-2187, Dec 2017.

[15] M. A. G. de Brito, L. Galotto, L. P. Sampaio, G. d. A. e Melo, and C. A. Canesin, "Evaluation of the main mppt techniques for photovoltaic applications," IEEE Trans. Ind. Electron., vol. 60, no. 3, pp. 1156-1167, March 2013.

[16] A. Safari and S. Mekhilef, "Simulation and hardware implementation of incremental conductance mppt with direct control method using cuk converter," IEEE Trans. Ind. Electron., vol. 58, no. 4, pp. 1154-1161, April 2011.

[17] "HSTBF24300P datasheet," Available at: http://www.solarpowercare.com, last accessed on July 2018.

[18] R. W. Erickson and D. Maksimovic, Fundamentals of power electronics. Springer Science \& Business Media, 2007. 